Check for updates

Cite this: Phys. Chem. Chem. Phys., 2020, 22, 20732

Received 16th April 2020

Accepted 17th June 2020

DOI: 10.1039/d0cp02053b

rsc.li/pccp

\title{
Photochemical and electrochemical reduction of graphene oxide thin films: tuning the nature of surface defects $\dagger$
}

\author{
Javier A. Quezada Renteria, (D) ab Cristina Ruiz-Garcia, (D) ${ }^{a}$ Thierry Sauvage, ${ }^{a}$ \\ Luis F. Chazaro-Ruiz, (iD b Jose R. Rangel-Mendez (iD ${ }^{b}$ and Conchi O. Ania (DD *a
}

\begin{abstract}
Individual and combined photo(electro)chemical reduction treatments of graphene oxide thin films have been performed to modulate the type of defects introduced into the graphene sheets during the reduction. These were characterized by X-ray photoelectron and Raman spectroscopies, nuclear reaction analysis and electrochemical methods. Illumination of the graphene oxide thin film electrodes with low irradiance simulated solar light provoked the photoassisted reduction of the material with negligible photothermal effects. The photoreduced graphene oxide displayed a fragmented $\mathrm{sp}^{2}$ network due to the formation of a high density of defects (carbon vacancies) and the selective removal of epoxides and hydroxyl groups. In contrast, the electrochemical reduction under mild polarization conditions favored the formation of $\mathrm{sp}^{3}$ defects over vacancies, with a preferential removal of carbonyl and carboxyl groups over hydroxyl/epoxides. Used in conjunction, mild photochemical and electrochemical treatments allowed the obtainment of reduced graphene oxides with varied reduction degrees (ca. C/O ratio ranging from 4.9 to 2.2), and surface defects. Furthermore, the electrochemical reduction prevented the formation of vacancies during the subsequent illumination step. In contrast, both types of defects were accumulated when the GO electrode was first exposed to illumination and then polarized.
\end{abstract}

\section{Introduction}

Graphene oxide (GO) has become one of the most affordable precursors to produce graphene-derived materials on a large scale. It can be prepared from bulk graphite by wet chemical oxidation and further exfoliation using relatively cost-effective (but time consuming) methods. GO has also unique properties due to its hydrophilic character and high chemical reactivity, owing to the oxygen-containing functional groups present in the carbon matrix. ${ }^{1}$ These oxygen groups can be removed to obtain partially reduced graphene oxide (rGO), a material with intermediate properties compared to GO and graphene. ${ }^{2}$ Even though plenty of methodologies have been applied to reduce

\footnotetext{
${ }^{a}$ CEMHTI, CNRS (UPR 3079), Université d'Orléans, 45071 Orléans, France.

E-mail: conchi.ania@crnrs-orleans.fr; Tel: +33-(0)238255513

${ }^{b}$ Division de Ciencias Ambientales, Instituto Potosino de Investigación Científica y Tecnológica A.C., camino a la presa san josé 2055, col. lomas 4 ㅇ sección, C.P. 78216, San Luis Potosí, Mexico

$\dagger$ Electronic supplementary information (ESI) available: Characterization of pristine GO (Table S1, Fig. S1 and S2); evolution of temperature during photochemical reduction (Fig. S3); XPS deconvolutions of C1s and O1s spectra (Fig. S5 and S7); NRA data (Fig. S6 and Table S2); Raman deconvolutions and fitting parameters (Fig. S8 and Table S3). See DOI: 10.1039/d0cp02053b
}

the surface groups in GO (e.g., chemical, ${ }^{3,4}$ thermal $^{5-8}$ and electrochemical $\left.^{9-11}\right)$, knowledge on the mechanisms still remains quite uncertain, as defects of different nature may be formed depending on the applied method and/or the nature of the groups. For instance, chemical and thermal reduction methods typically render very reactive rGO materials with a high density of edge-like zones (vacancies or holes). ${ }^{5-7,12,13}$ Besides defects, the chemical reduction may also result in the incorporation of heteroatoms from the reducing agent (e.g., $N$-functionalization using hydrazine) ${ }^{3} \quad$ Electrochemical methods seem to favor the removal of epoxides, phenolic and carbonyl groups over carboxylic moieties depending on the electrolytic medium. ${ }^{9-11}$ Photochemical treatments have also been investigated to obtain highly reduced graphene oxides, mainly through photothermal effects with intense light sources (e.g., UV light ${ }^{14}$ or high power lasers ${ }^{15}$ ) and often in the presence of a catalyst. Some attempts have also been reported on the use of solar light for a mediated reduction of GO, rendering rGO with less structural defects and higher conductivity than the material obtained by chemical reduction. ${ }^{16}$ While most studies focus on the evolution of O-groups upon reduction treatment, less attention is paid to the nature and/or location of the structural defects introduced into the rGO as a 
result of de-functionalization. These aspects are important to understand the different physicochemical characteristics of rGO (e.g., optical, structural, and reactivity) compared to graphene, as they govern its reactivity and performance. ${ }^{17-19}$

Aiming to modulate the type of surface defects introduced into the graphene sheets of rGO materials, this work describes the photochemical and electrochemical reduction of GO thin films in aqueous medium under mild conditions. By applying individual and combined photo-/electrochemical treatments, different types of defects were incorporated into the resulting rGO films. The photochemical reduction under simulated solar light favored the removal of epoxides and hydroxyl groups, rendering $\mathrm{rGO}$ with fragmented $\mathrm{sp}^{2}$ domains and a high density of structural defects with predominance of carbon vacancies. In contrast, the electrochemical reduction favored the formation of $\mathrm{sp}^{3}$ defects over vacancies. When both processes were combined, the electrochemical reduction prevented the formation of vacancies during the subsequent illumination step. However, when the order was reversed (i.e., photochemical treatment followed by electrochemical reduction), both types of defects were accumulated.

\section{Results and discussion}

\section{Characterization of the bulk GO material}

Graphene oxide used for the preparation of thin film electrodes was obtained by oxidation of graphite powders using the Hummers' method. The as-prepared bulk GO was characterized to determine the characteristics of the initial material before the reduction treatments. Data from elemental analysis (Table S1, ESI $\dagger$ ) and temperature programmed desorption coupled to mass spectrometry (Fig. S1, ESI $\dagger$ ) confirmed the large oxidation extent of the prepared GO (ca. $46 \mathrm{wt} \% / 33$ at\% oxygen). The characteristic profiles of $\mathrm{CO}$ and $\mathrm{CO}_{2}$ gases evolved from the decomposition of the O-containing groups also confirmed the high oxidation state of the prepared graphene oxide. The sharp decomposition peaks for $\mathrm{CO}$ and $\mathrm{CO}_{2}$ at temperatures around $200{ }^{\circ} \mathrm{C}$ are attributed to the decomposition of epoxides and hydroxyl groups. This is in agreement with TPD studies performed for graphene oxides in the literature, ${ }^{2,20-25}$ also reporting the difficulty to differentiate between both moieties decomposing at close temperatures. The small contribution for $\mathrm{CO}_{2}$ evolution at $300-450{ }^{\circ} \mathrm{C}$ indicates the decomposition of carboxylic acid groups, and the CO evolution above $450{ }^{\circ} \mathrm{C}$ is characteristic of the decomposition of phenols and carbonyl/quinones. The higher contribution of epoxides and hydroxyl groups over quinones and carboxylic acids was also confirmed by X-ray photoelectron spectroscopy (XPS) analysis (Table 1), as it will be discussed below. The characteristics of the synthesized bulk GO are in good agreement with those reported in the literature for graphene oxides prepared following a similar procedure. ${ }^{2,22,26}$ The XRD diffractograms of graphite and bulk GO revealed that the $(002)$ reflection shifted from $26.3^{\circ}(0.34 \mathrm{~nm})$ for the starting graphite to $11.2^{\circ}(0.78 \mathrm{~nm})$ for the prepared GO (Fig. S2, ESI $\dagger$ ). This represents a two-fold increase in the distance between the graphene layers of graphite upon oxidation and exfoliation.

\section{Preparation of the rGO thin film electrodes}

In a typical photo-/electrochemical reduction experiment, the GO thin films deposited on the ITO substrate were placed in an aqueous electrolyte and illuminated and/or polarized as indicated in the Experimental section. It is important to remark that since our study focuses on the control of the defects

Table 1 Concentration of elements (at\%) and relative abundance (\%) of the different peaks assigned from the deconvolution of the C1s and O1s core energy levels of the thin film electrodes

\begin{tabular}{|c|c|c|c|c|c|}
\hline (at\%) & C & $\mathrm{O}$ & $\mathrm{N}$ & $\mathrm{S}$ & Ratio $\mathrm{C} / \mathrm{O}$ \\
\hline GO & 61.5 & 34.6 & 1.9 & 2 & 1.8 \\
\hline ErGO & 66.8 & 29.7 & 1.8 & 1.7 & 2.2 \\
\hline PhrGO & 70.3 & 28.0 & 1.7 & $<0.1$ & 2.5 \\
\hline PhErGO & 72.5 & 24.6 & 2.7 & 0.2 & 2.9 \\
\hline EPhrGO & 81.9 & 17.5 & 0.5 & $<0.1$ & 4.7 \\
\hline
\end{tabular}

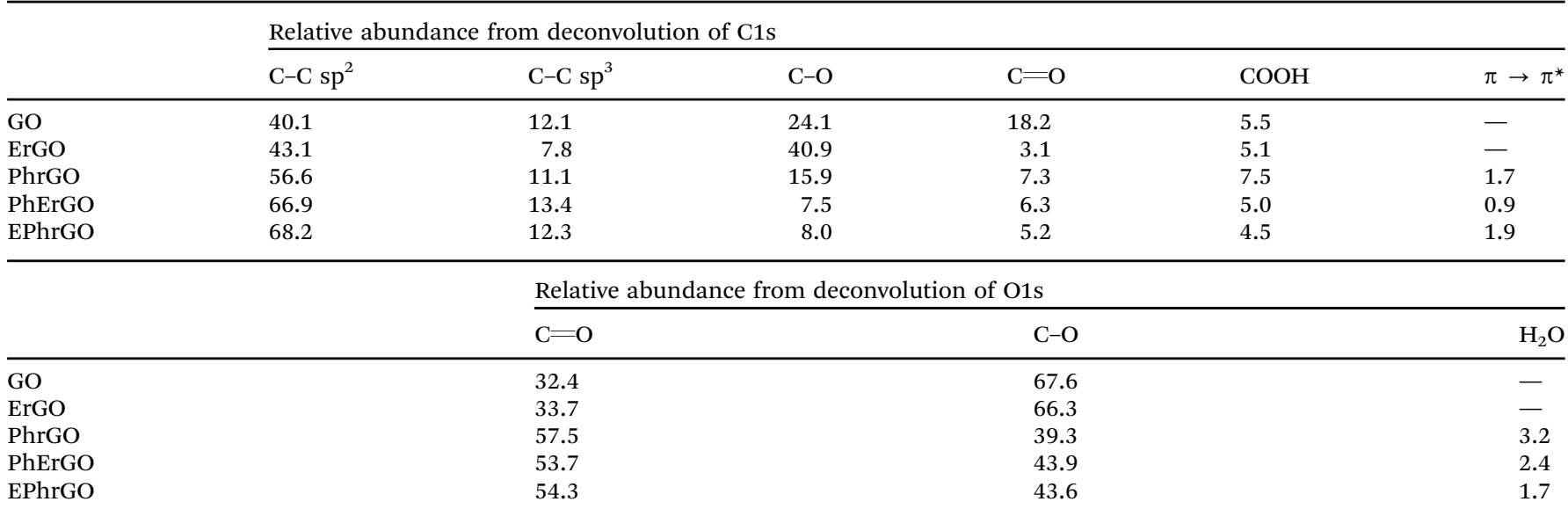


created on the rGO electrodes, mild electrochemical and photochemical reduction conditions were applied. Thus, the photoelectrochemical treatments were carried out under ambient conditions on non-deaerated electrolytic solution. This implies that dissolved oxygen is present during the reduction, and that it might be contributing to the eventual reoxidation of the graphene sheets. This would account for the relatively low reduction degree of the obtained rGO materials.

The electrochemical reduction of the electrodes was carried out by cyclic voltammetric scanning in the range from -1 to $+0.6 \mathrm{~V}$ ( $v s . \mathrm{Ag} / \mathrm{AgCl}$ ) as indicated in the Experimental section. These are mild electrochemical conditions compared to the high voltages $(c a .20 \mathrm{~V}),{ }^{27}$ and acidic/basic electrolytes ${ }^{28,29}$ commonly applied to obtain rGO with a high reduction degree. In the photochemical reduction experiments, the GO electrode was illuminated for 6 hours using simulated solar light (unfiltered) to obtain sample PhrGO. The lamp source emitted in a $3 \mathrm{~cm}$ diameter area, and it was positioned at $80 \mathrm{~mm}$ distance from the electrode immersed in the electrolyte. This allowed the complete illumination of the geometrical area of the electrodes. The light intensity delivered at the surface of the electrodes was $c a .0 .15 \mathrm{~W} \mathrm{~cm}^{-2}$, much lower than common values (ca. 1-1.2 $\mathrm{W} \mathrm{cm}^{-2}$ ) reported for photochemical and photothermal reductions. ${ }^{30,31}$ It should be mentioned that the temperature of the electrolytic solution during the illumination of the electrodes increased from 25 to $31{ }^{\circ} \mathrm{C}$ within the first $60 \mathrm{~min}$ (an increase from 25 to $27^{\circ} \mathrm{C}$ was measured upon illumination of the electrolyte alone, Fig. $\mathrm{S} 3$, $\mathrm{ESI} \dagger)$. This is important to differentiate between photochemical and photothermal conversion; the latter is based on the decomposition of the O-groups as a result of the local increase in the temperature upon irradiation, and it is usually reported when intense light sources (in terms of energy flux) are used; this will be further discussed below.

Fig. 1 shows the voltammetry scanning curves of the reduced thin film electrodes and the response of the pristine GO thin film electrode is shown as control. The first voltammetry scan corresponds to the electrochemical behavior of the given sample itself (e.g., pristine GO) and the subsequent scans correspond to the electrochemical reduction (e.g., scans of GO to yield ErGO; or PhrGO to yield PhErGO). As seen, a very small capacitive current was recorded for the first cycle of the GO thin film electrode on the anodic part of the voltammetric response; this is characteristic of materials with poor electrical conductivity as GO. Subsequent voltammetric scans in GO are not reversible, indicating that the material is modified (electroreduced) during the cycles; the second and following scans brought about a gradual increase in the current and the appearance of a broad quasi-reversible response. A cathodic hump between -0.4 and $-0.2 \mathrm{~V}$ and an anodic peak barely perceptible at $-0.1 \mathrm{~V}$ point out to a pseudocapacitive redox process (typical potential ranges of the quinone/hydroquinone pair). ${ }^{32}$ The cathodic current increasing from $-0.8 \mathrm{~V}$ in the $\mathrm{GO}$ is related to the incipient decomposition of the electrolyte; this cathodic current leap gradually moved towards more negative potential values with the voltammetric scans, which is characteristic of a more reduced graphene oxide material. ${ }^{28,33}$ After 10 scans, the voltammetric response of GO became reversible with no further
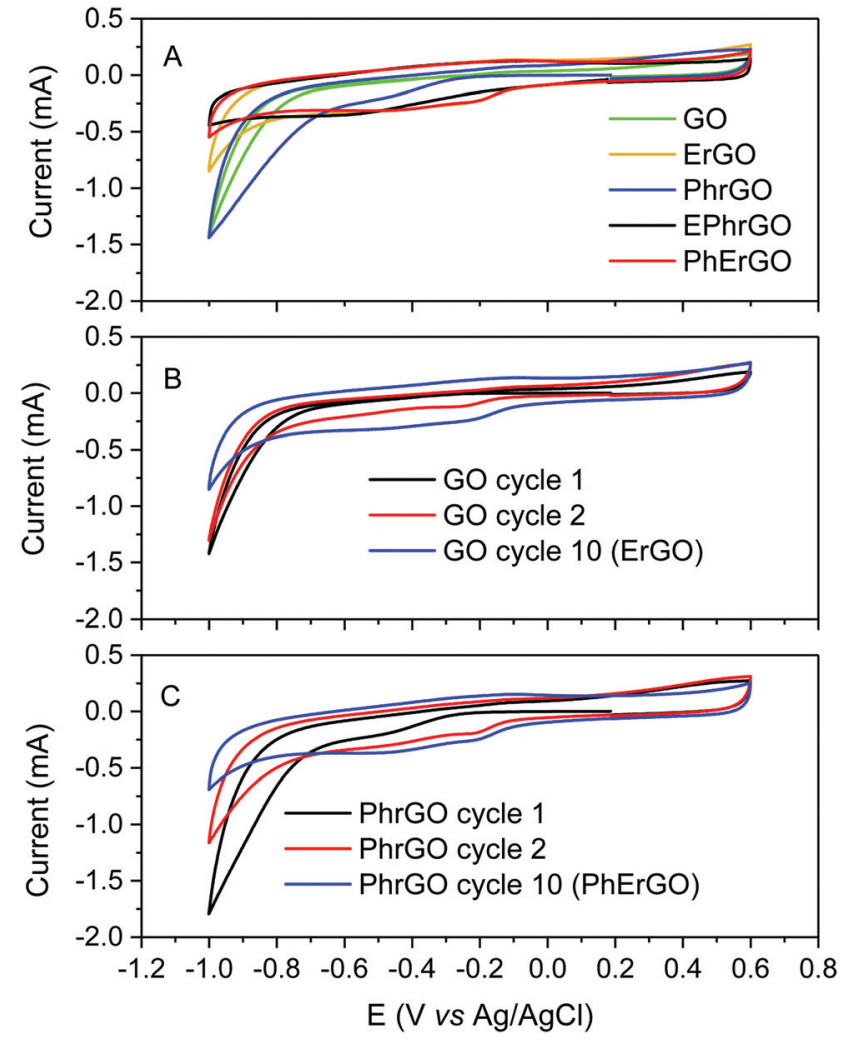

Fig. 1 Cyclic voltammograms recorded at $2 \mathrm{mV} \mathrm{s}^{-1}$ in $0.6 \mathrm{mM} \mathrm{NaCl}$ electrolyte for the series of thin film electrodes supported on the ITO glass substrate: (A) first cycle of each electrode; (B) selected cycles on the GO electrode to obtain ErGO; (C) selected cycles on the PhrGO electrode to obtain PhErGO.

changes in the shape, intensity and peak potentials (Fig. S4, ESI $\dagger$ ). This indicates that no further electrochemical reduction occurs upon cycling in this scanning potential range. The sample after 10 polarization cycles is then labelled as ErGO.

For sample PhrGO, the initial voltammetric scans are also not reversible, showing a rapid increase in the cathodic current and a wide peak at $-0.5 \mathrm{~V}$. This indicates that sample PhrGO is further electrochemically reduced upon cycling. Similar results on a slow photoassisted reduction mechanism have been reported for GO exposed to illumination between 6 and 48 hours under more energetic conditions. ${ }^{14}$

To evaluate the extent of the electrochemical reduction of PhrGO, the sample was subsequently polarized under similar conditions to sample ErGO to obtain sample PhErGO. Similarly, the electroreduced ErGO electrode was also exposed to $6 \mathrm{~h}$ of illumination to prepare sample EPhrGO. The voltammetric response of EPhrGO showed a capacitive response close to that of ErGO (Fig. 1A); subsequent cycles overimposed to the first one, indicating a stable electrochemical behavior (also observed in ErGO). In contrast, the polarization cycles of PhrGO to render PhErGO showed an increasing current during the first cycles (Fig. S4, ESI $\dagger$ ), becoming stable after the 10th scan and evidencing a more reduced state of PhErGO.

Based on the voltammetric responses of all four electrodes, it seems that the photochemical reduction takes place to a 


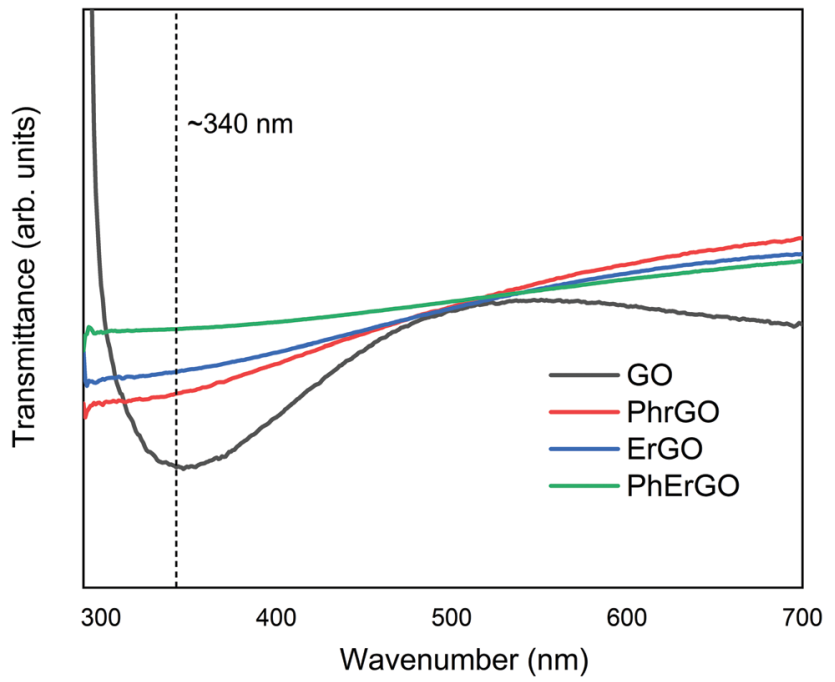

Fig. 2 UV-vis spectra of the series of GO and rGO thin film electrodes cast on the ITO glass substrate.

minor extent than the electrochemical one. Illumination of the electrochemical reduced electrode does not seem to modify much the electrochemical performance of the material. This contrasts with the differences observed in the O-groups remaining in the electrodes after the reduction treatments, as determined by XPS analysis (see discussion below).

Changes due to the reduction of the electrodes are also noted in their optical and light absorption features. The transparent brownish color characteristics of GO cast on the ITO substrate changed progressively to opaque dark brown/black upon illumination and polarization, due to the removal of O-groups. These changes were also monitored by UV-vis absorption spectroscopy of the electrodes (Fig. 2). Due to the cut-off threshold of the glass substrate used as support for the electrodes, the spectra can only be recorded above $300 \mathrm{~nm}$ (i.e., the absorption peak at ca. $233 \mathrm{~nm}$ corresponding to the $\pi-\pi^{*}$ transition in conjugated C-C domains cannot be observed). The typical $n-\pi^{*}$ transition peak of carbonyl moieties at around $300 \mathrm{~nm}$ is clearly seen for the pristine GO electrode. ${ }^{34}$ The intensity of this peak decreased for the illuminated and polarized electrodes, which can be considered as an indicator of the reduction of the material. The decrease was more pronounced for the electrodes that were exposed to both lighting and polarization, indicating a greater loss of carbonyl, quinones or carboxylic groups. This is in agreement with the characterization of the rGO electrodes by XPS and NRA techniques (see discussion below). Although the use of light and electrochemical methods for the reduction of GO has been investigated, ${ }^{35-37}$ our data show the strong impact of the use of mild photochemical and electrochemical conditions for the selective removal of O-groups and the creation of different types of surface defects.

\section{Chemical characterization of the thin film electrodes}

The electrodes were further characterized by XPS, Raman spectroscopy and nuclear reaction analysis (NRA) to explore the impact of photochemical and electrochemical reduction approaches in the structural and chemical features of the materials. The low thickness of the films (between 100 and $300 \mathrm{~nm}$ as estimated by NRA, Table S2, ESI $\dagger$ ) along with the cut-off temperature and absence of crystallinity of the glass substrate prevented the use of XRD and TPD for bulk characterization of the thin film electrodes after the reduction. Thus, the chemical composition and speciation of the surface groups were explored using XPS and NRA. The starting electrode prepared by casting GO on the ITO support was also characterized for comparison purposes.

Fig. 3 shows the XPS spectra of the C1s region of the thin film electrodes and the surface atomic percentage is summarized in Table 1. The characterization of the GO electrode revealed a high percentage of oxygen in the material (ca. 35 at\%), in agreement with the composition of bulk GO determined from elemental analysis ( $c a .33$ at\%, Table S1, ESI $\dagger$ ). Besides oxygen, small amounts of nitrogen and sulfur were detected (sodium nitrate and sulfuric acid are used in the Hummers' method to prepare the GO).

The XPS spectra of the C1s region were deconvoluted into various contributions at binding energies of $284.6 \mathrm{eV}\left(\mathrm{Csp}^{2}\right)$, $285.4 \mathrm{eV}\left(\mathrm{Csp}^{3}\right), 286.2 \mathrm{eV}$ (hydroxyl and epoxide groups, denoted as $\mathrm{C}-\mathrm{O}$ ), $287.3 \mathrm{eV}$ (ketones, aldehydes and quinones,
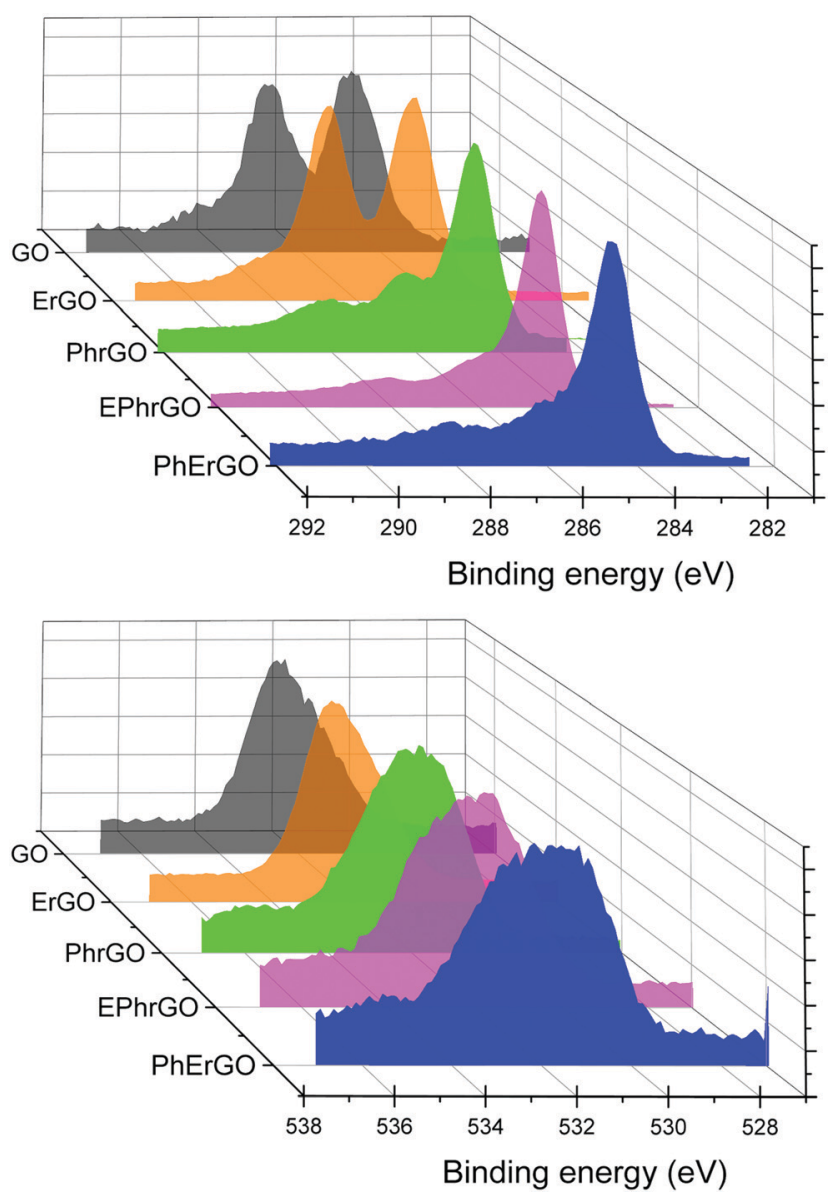

Fig. 3 High resolution XPS C1s (top) and O1s (down) core level spectra of the pristine and reduced thin film electrodes. 
denoted as $\mathrm{C}=\mathrm{O}$ ), $288.4 \mathrm{eV}$ (carboxylic acids, anhydrides and esters, denoted as $\mathrm{COOH})$, and $290.3 \mathrm{eV}\left(\pi-\pi^{*}\right.$ shake-up satellite peak). ${ }^{38,39}$ Full deconvolutions are shown in Fig. S5 (ESI $\dagger$ ). The large contribution of the peak at $286.2 \mathrm{eV}$ for the GO electrode is indicative of a large amount of epoxide and hydroxyl groupscompared to carbonyl and carboxylic groups-. This agrees with the large peak evolving at $c a .200{ }^{\circ} \mathrm{C}$ in the TPD profiles of the bulk material (Fig. S1, ESI $\dagger$ ), as discussed above. It also points out the stability of GO during the fabrication of thin film electrodes.

XPS data confirmed the removal of the O-groups in the reduced electrodes, with important differences among the samples. As shown in Table 1, the amount of surface oxygen decreased smoothly with different treatments, following the order: GO $>$ ErGO $>$ PhrGO $>$ PhErGO $>$ EPhrGO. The relatively large amounts of oxygen remaining in the resulting rGO are associated with the mild reduction conditions, and with the partial reoxidation involving dissolved oxygen as the treatments were carried out in aerated solutions. Nonetheless, the samples display different types of structural defects, as it will be discussed below. The quantification of the carbon and oxygen contents by NRA renders a similar C/O ratio to XPS, ranging from 1.9 to 4.6 (Table S2 and Fig. S6, ESI $\dagger$ ). Furthermore, NRA spectra show no significant oxygen concentration gradient in the films with a depth resolution of $15 \mathrm{~nm}$. The good agreement with the data from XPS indicates that the reduction is homogeneous over the surface of the thin film electrodes (since NRA probes the volume of the film and is representative of $2 \times 2 \mathrm{~mm}^{2}$, whereas XPS is a surface analysis technique and probes a few upper nm).

The deconvolution of the C1s spectra also revealed important differences in the nature of the groups remaining in the materials after the electrochemical and photochemical reduction. As shown in Fig. 3, the contribution of carbon in an oxidized state decreased in all the rGO electrodes compared to pristine GO, with the smallest impact observed for sample ErGO. For instance, sample PhrGO presented almost twice lower surface oxygen than pristine GO, while sample ErGO still presents a high oxygen content. Furthermore, data show that the photochemical reduction selectively removed the epoxide/hydroxyl groups present in GO (ca. $70 \%$ fall in the $\mathrm{C}-\mathrm{O}$ signal at $286 \mathrm{eV}$ ), whereas the electrochemical reduction brought about only a $10 \%$ decrease in these groups (Table 1 and Fig. 3). These results contrast with the electrochemical features of the electrodes determined by cyclic voltammetry (Fig. 1) that showed a better conductivity in ErGO compared to PhrGO. Thus, it seems that despite its lower oxygen content, the groups remaining on PhrGO (mainly carbonyl and carboxylic acids) are responsible for the poor electrochemical response. Given the differences in the relative abundance of O-groups of both samples, this behavior must be attributed to the higher fraction of carboxylic groups (Table 1), which are known to have a high electron withdrawal character. It is also worth noting that the signal associated with the $\pi-\pi^{*}$ shake-up satellite peak was not detected for GO and ErGO (Fig. 3), but started to be notable for samples PhrGO, PhErGO and EPhrGO. This evidences the presence of conjugated aromatic domains in the carbon lattice in the latter reduced samples. Similar findings have been reported for the hydrothermal reduction of GO suspensions. ${ }^{40}$

For the samples exposed to both electrochemical and photochemical treatment, the contribution of the peaks associated with oxidized carbon decreased considerably. The reduction was more efficient in sample EPhrGO, which presented the lowest oxygen content (Table 1 ). The higher intensity of the peak associated with the $\pi^{*}$ shake-up satellite peak indicates the presence of electronically conjugated domains of larger size as a consequence of a higher reduction extent. ${ }^{36,37}$ Data from the O1s spectra followed a similar trend (Table 1; full deconvolutions of O1s spectra are shown in Fig. S7, ESI $\dagger$ ). The fraction of epoxide and hydroxyl groups decreased gradually, with a larger relative abundance of quinone/carboxylic moieties in the samples exposed to both reduction treatments.

\section{Structural characterization of the thin film electrodes}

The structural changes of the pristine and reduced electrodes were analyzed by Raman spectroscopy (Fig. 4). Data between 1000 and $1750 \mathrm{~cm}^{-1}$ were deconvoluted showing the characteristic $\mathrm{G}$ (around $1580 \mathrm{~cm}^{-1}$ ) and D (around $1350 \mathrm{~cm}^{-1}$ ) bands of graphite and derived carbon materials in the first order region. To better fit this spectral region, different contributions of bands $\mathrm{D}^{\prime}$ (around $1600 \mathrm{~cm}^{-1}$ ), $\mathrm{D}^{\prime \prime}$ (around $1506 \mathrm{~cm}^{-1}$ ) and $\mathrm{D}^{*}$ (around $1220 \mathrm{~cm}^{-1}$ ) were introduced. ${ }^{41-43}$ They correspond to disorder in the surface, out of plane defects, and disorder graphitic lattices and impurities, respectively ${ }^{45}$ (all deconvoluted spectra and parameters are shown in Fig. S8 and Table S3, $\mathrm{ESI} \dagger)$. The presence of these bands and their relative positions, widths and intensities provide information about the existence and nature of surface defects in graphene-like materials. ${ }^{19,44-50}$

The position of the $\mathrm{G}$ band ( $c a .1580 \mathrm{~cm}^{-1}$ ) remained unchanged for all the samples, but its width increased significantly for the reduced electrodes, regardless of the treatment. The broadening of the $\mathrm{G}$ band is indicative of the presence of structural defects in the $\mathrm{sp}^{2}$ network of the reduced samples (i.e., smaller defect-free graphene-like domains). ${ }^{45}$ The prominent $\mathrm{D}$ band ( $c a .1350 \mathrm{~cm}^{-1}$, intervalley Raman mode) in the GO arises from the imperfections created by the attachment of the O-groups on the carbon basal plane, ultimately indicating a relative measure of the amount of $\mathrm{sp}^{3}$ carbons and vacancies. ${ }^{46,47}$ Although the frequency of the D mode is almost insensitive to the origin and kind of defects in carbon materials, its intensity is typically used as an indicator of their density. ${ }^{48}$ In the case of reduced samples, the $\mathrm{D}$ band became narrower and its intensity decreased; this implies a partial restoration of the $\mathrm{sp}^{2}$ hybridization state of the carbon crystalline lattice. The disorderinduced broadening of the $\mathrm{G}$ band was accompanied by the appearance of the $\mathrm{D}^{\prime}$ band, associated with the disorder defects such as impurity atoms, and folding or double vacancies in pentagonal and octagonal rings (i.e., 5-8-5 defects). ${ }^{43,49}$

The band $\mathrm{D}^{\prime \prime}$, assigned to amorphous carbon lattices, followed an opposite trend of becoming narrower for the reduced samples: GO $>$ PhrGO $>$ ErGO $>$ PhErGO $>$ EPhrGO. This indicates a lower number of the out-of-plane defects (e.g., $\mathrm{sp}^{3}$ atoms in the 

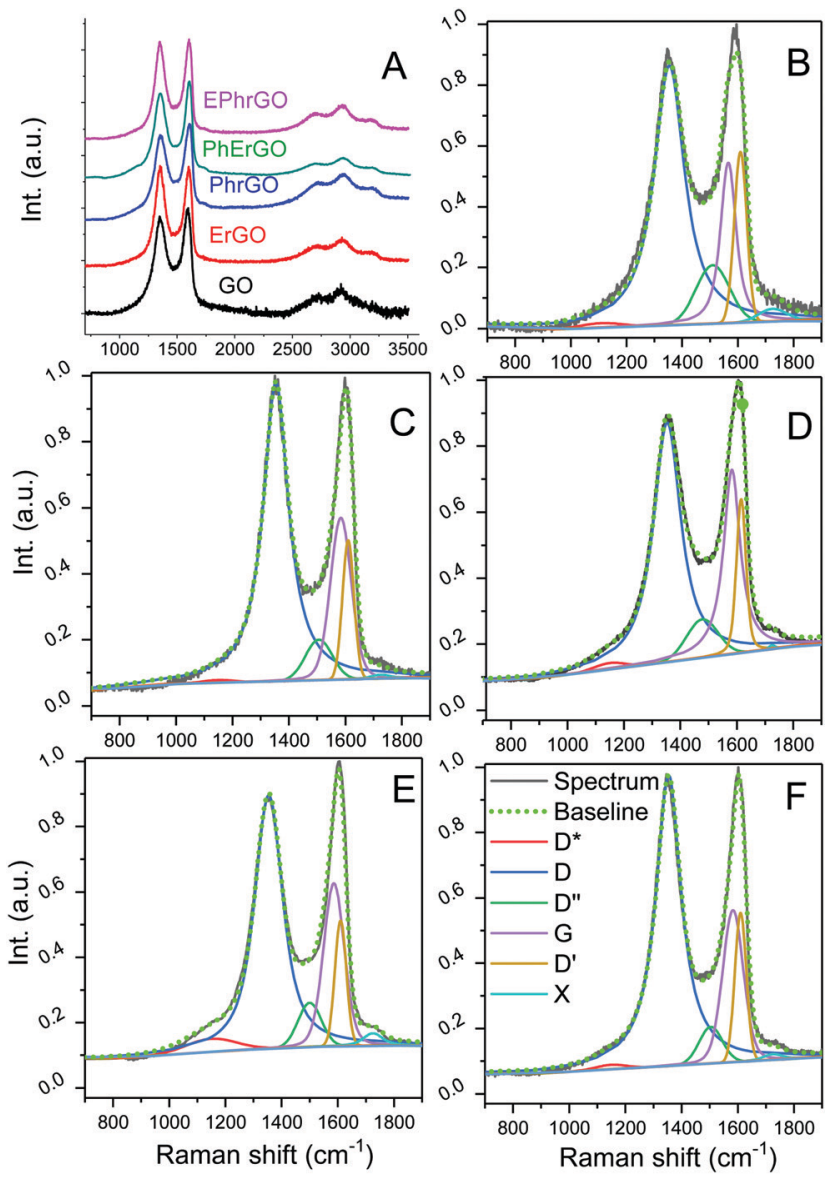

Fig. 4 Raman spectra of the studied electrodes (A); deconvolution of first-order spectra of (B) pristine GO, (C) PhrGO (D) ErGO, (E) PhErGO and (F) EPhrGO.

$\mathrm{sp}^{2}$ network) compared to GO and is in agreement with the low amount of hydroxyl and epoxide groups (responsible for the $\mathrm{sp}^{3}$ hybridization in the $\mathrm{sp}^{2}$ aromatic structure of the graphene sheets) in the reduced samples. ${ }^{45}$ This trend would explain the different electrochemical responses of the electrodes (Fig. 1), with a poor capacitive response of sample PhrGO compared to more oxidized ErGO. This is an indicator of the different introduction of defects during the elimination of the O-groups, as it will be discussed ahead.

All the studied materials presented $I_{\mathrm{D}} / I_{\mathrm{G}}$ values lower than 3.5 , thus they can be assimilated to materials with a low defect degree (stage 1) according to the terminology of graphenic materials introduced by Ferrari et al. ${ }^{47}$ The evolution of the $I_{\mathrm{D}} / I_{\mathrm{G}}$ values goes from 1.6 for pristine $\mathrm{GO}$ to the following trend: 1.3 (PhrGO), 1.6 (PhErGO), 1.8 (ErGO), and 1.9 (EPhrGO).

For stage 1 graphenic materials, the intensities of the $\mathrm{D}$ and $\mathrm{D}^{\prime}$ bands are proportional to each other and proportional to the defect concentration and to the types of defects, grain boundaries, vacancies, or $\mathrm{sp}^{3}$ hybridization. The dependence between $I_{\mathrm{D}}$ and $I_{\mathrm{D}^{\prime}}$ followed a linear trend (Fig. $5 \mathrm{~B}$ ): PhrGO $>$ GO $>$ EPhrGO $>$ PhErGO $>$ ErGO. This indicates that the density of defects is higher in PhrGO and lower in ErGO; regarding the combined photochemical and electrochemical reduction, there are more defects when the photochemical treatment is carried out second (sample EPhrGO). Recent studies based on experimental and theoretical calculations have proposed that it is possible to discriminate the presence of vacancies and $\mathrm{sp}^{3}$ defects upon the correlation between the areas of the $\mathrm{D}^{\prime}$ and $\mathrm{D}$ bands (normalized $v s$. the $\mathrm{G}$ band), defining two differentiated zones. ${ }^{19,49-51}$ This correlation is shown in Fig. 5A for our samples compared to data from the literature. ${ }^{19}$

Our results show that sample PhrGO belongs to the first group where vacancy-like structural defects are predominant, while samples ErGO and EPhrGO belong to the second group where defects associated with $\mathrm{sp}^{3}$ hybridization are predominant. The samples exposed to both photochemical and electrochemical reduction treatments displayed a mixed character (particularly sample PhErGO), indicating that the vacancies are first created upon photochemical reduction, and the subsequent reduction during the polarization cycles creates other type of defects. However, the correlation between ID and ID' (Fig. 5B) suggests that there are more defects when the photochemical treatment is carried out second (sample EPhrGO) compared to when the process is reversed. This would suggest that the vacancies created in the first photochemical treatment would be restored or converted to other defects during the second electrochemical reduction (sample PhErGO). With the exception of sample EPhrGO, neither the $\mathrm{Csp}^{2}$ nor the $\mathrm{Csp}^{3}$ contents in the reduced samples followed a linear correlation with the width of the D band (Fig. 5C and D), indicating that only a fraction of the eliminated functional groups lead to the restoration of carbon $\mathrm{sp}^{2}$ bonds. Thus we can infer that the samples present a high density of defects related to vacancies.

The hump in the Raman spectra appearing at $c a .1700 \mathrm{~cm}^{-1}$ (assigned as $\mathrm{X}$ in Fig. 4) has a frequency considerably lower ( $c$ a. $50 \mathrm{~cm}^{-1}$ ) than the $\mathrm{M}$ band featuring in bilayer and few layer graphenes. ${ }^{52}$ Despite being a common feature in the Raman spectra of carbon materials, it is often disregarded; its interpretation is not clear, although it has been proposed that is related to non-regular rings.

It should also be mentioned that Raman peaks are sensitive to structural/topological defects in $\mathrm{Csp}^{2}$ regions in graphenic materials, while $\mathrm{Csp}^{3}$ regions are not detected. This explains why materials with different $\mathrm{Csp}^{2}$ contents may display similar D bands (samples ErGO and PhrGO), accounting for different structural defects $\left(\mathrm{sp}^{3}\right.$, ripples, vacancies, and roughness).

In the second-order region of the spectra, all the samples showed similar features, with a low intensity of the $2 \mathrm{D}$ band and the broad bands from 2300 to $3100 \mathrm{~cm}^{-1}$ assigned to $2 \mathrm{D}^{\prime}$ and combination bands. The contributions of the latter bands are less important for the samples with the highest reduction degree (i.e., lowest oxygen content), indicating the broken symmetry of the graphene sheets in the samples as a result of the presence of oxygen groups and defects.

\section{Proposed mechanism for the photo-/electrochemical reduction of the thin film electrodes}

Most studies on the photoreduction of GO suspensions report that the reaction is triggered by the electron transfer from a 

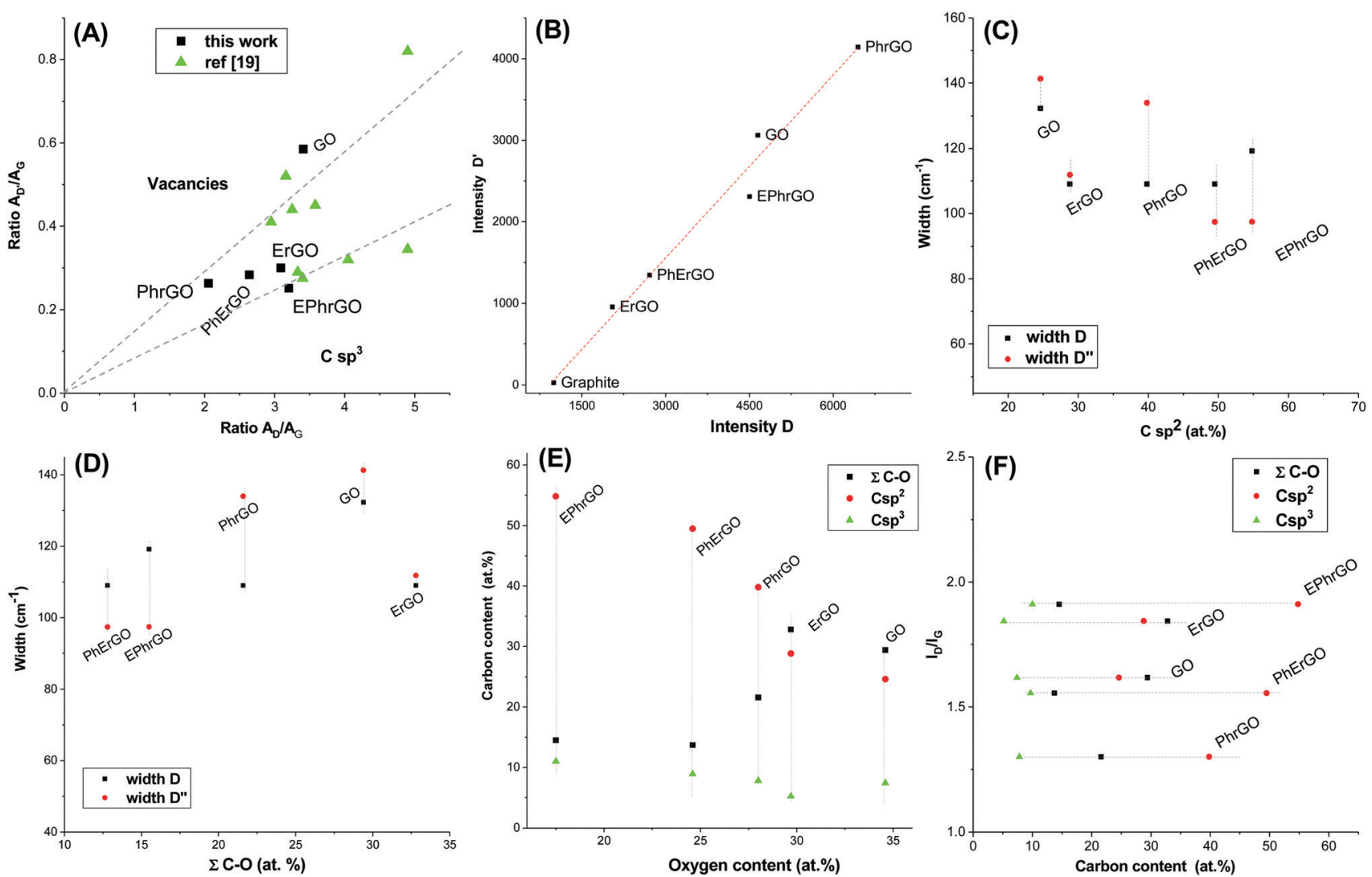

Fig. 5 Correlations between structural (Raman) and composition (XPS) features of the samples: (A) ratio $A_{D^{\prime}} / A_{G} v s$. $A_{D} / A_{G} ;(B) I_{D^{\prime}} v s$. $I_{D} ;$ width of bands $D$ and $D^{\prime \prime}$ vs. (C) $\mathrm{Csp}^{3}$ content and vs. (D) C-O content; (E) carbon content vs. oxygen content; (F) $I_{D} / I_{G} v s$. carbon content. Lines are a guide for the eye.

sacrificial agent to the GO sheets ${ }^{53,54}$ (catalyzed or not) or due to the photothermal mechanism for powerful light sources (e.g., pulsed lasers). ${ }^{30,31,55}$ Under our experimental conditions, photothermal effects cannot be considered as the origin for the removal of the O-groups, since the temperature increase only represented $\Delta T \sim 4-5{ }^{\circ} \mathrm{C}$ (Fig. S3, ESI $\dagger$ ). Such a temperature increase is not enough to activate direct thermal dissociation, demonstrating that only photochemical effects are relevant under our experimental conditions. This is consistent with the intensity delivered to the electrodes ( $c a .0 .15 \mathrm{~W} \mathrm{~cm}^{-2}$ ), one order of magnitude lower than the values typically reported for photothermal or photocatalytic reductions. ${ }^{30,31,55}$

Since GO is a good light absorber, photogenerated excitons are expected to be formed upon illumination produced from the $\pi-\pi^{*}$ and $n-\pi^{*}$ transitions. ${ }^{56}$ To investigate the mechanism of the photoassisted reduction under simulated solar light, we measured the open circuit potential (OCP) of the thin film electrodes upon on/off illumination cycles of $c a .10 \mathrm{~min}$ (Fig. 6). The illumination time was restricted to avoid the modification of the electrodes. Under dark conditions, the OCP represents the interfacial surface charge upon immersion in the electrolyte; when the electrodes are illuminated, surface charge redistribution is expected. For the GO thin film electrode, the dark OCP shifted ca. $20 \mathrm{mV}$ towards positive values upon on/off illumination.

The photopotential increase demonstrates the generation of electron-hole pairs on the GO surface after photon absorption,

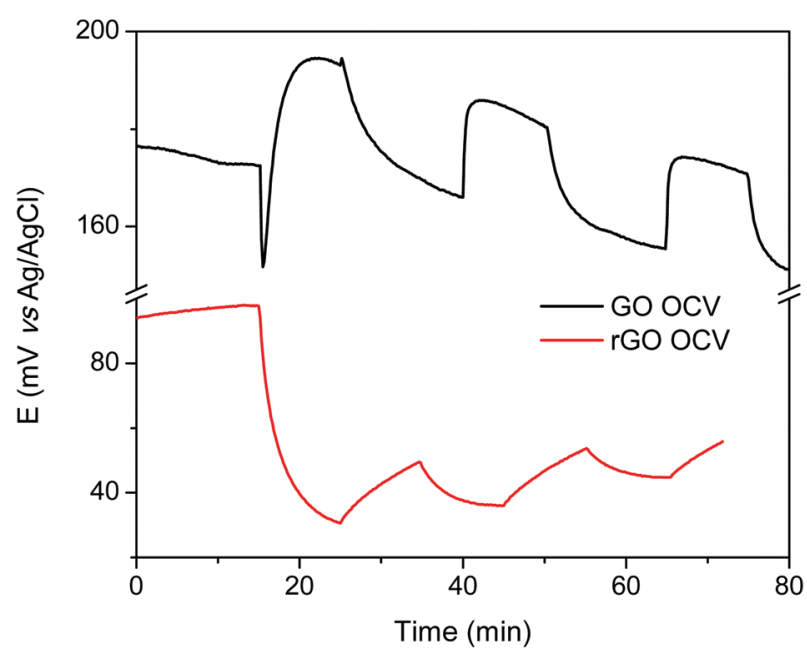

Fig. 6 Open-circuit potentials upon on/off illumination under simulated solar light of $\mathrm{GO}$ and $\mathrm{rGO}$ electrodes.

thereby generating an electric field that compensates the band bending in the dark. The fact that the potential shifted towards positive values is characteristic of p-type semiconductors, where the electrons generated upon irradiation are trapped and transferred to available species faster than the holes that accumulate (thereby shifting the OCP to a more positive potential). The photogenerated electrons at the GO interface 
can either be transferred to other species (e.g., dissolved oxygen, water, carbon dioxide, and electrolyte) or to the surface groups of the electrode material.

Based on the characterization of sample PhrGO, it is evident that the photogenerated electrons are responsible for the reduction of the GO and the removal of a large fraction of O-groups (see XPS data). It should be mentioned that the on/off illumination of the naked ITO substrate showed an opposite trend, with a decreased photopotential of $c a .20 \mathrm{mV}$, since it is an n-type semiconductor. Thus, the positive potential increase upon illumination of the GO cast on the ITO substrate can only be attributed to the photoresponse of the GO. This indicates that the GO thin film electrode acts as a p-type semiconductor upon illumination. Although several studies have reported similar observations, ${ }^{57,58}$ some others have described an n-type semiconductor response for GO electrodes. ${ }^{59,60}$ Indeed, the nature and origin of the optical transitions upon its illumination are still quite unclear, with rather contradictory observations; this may be attributed to different aspects including the extent of the oxidation of the pristine GO (see discussion below) and the conditions of the illumination of the electrodes (source, wavelength, power, and atmosphere).

A different photoelectrochemical response was observed after reduction. All the reduced samples showed a shift in the OCP upon on/off illumination towards negative values due to the accumulation of the photogenerated electrons $(c a .70 \mathrm{mV}$, larger that the OCP corresponding to the naked ITO substrate). This behavior confirms the n-type semiconducting character of the rGO electrodes (as opposed to that of GO), which is attributed to the presence of the conjugated $\mathrm{sp}^{2}$ domains in the reduced samples. For n-type rGO semiconductors, the photoholes are transferred to solution species faster than the photoelectrons, and consequently the OCP moves to a more negative potential. The photopotential profiles (particularly for rGO) also evidence a slow kinetics of the charge transfer across the electrode, with long times needed to reach equilibrium potentials. This is characteristic of materials with multiple surface states where the charge carriers are trapped (e.g., defects, O-groups). ${ }^{61}$

Based on the characterization of the reduced materials and the p-type semiconducting character of the starting GO, the photochemical reduction is expected to proceed through the removal of epoxide/hydroxyl groups upon reaction with the photogenerated holes to form $\mathrm{C}=\mathrm{C}$ double bonds and carbon vacancies. This reaction would be accompanied by the loss of carbon atoms in the form of $\mathrm{CO}$ and/or $\mathrm{CO}_{2}$ and affects mostly the epoxide/hydroxyl groups, leading to a material with fragmented $\mathrm{sp}^{2}$ domains with vacancies, summarized as:

$$
\begin{gathered}
\mathrm{C}-\mathrm{O}_{[\mathrm{GO}]}+\text { photoholes } \rightarrow \mathrm{C}=\mathrm{C}, \mathrm{C}_{\text {vacancy }}(\text { defect carbon })_{[\mathrm{rGO}]}, \\
\mathrm{CO}_{2} / \mathrm{CO}
\end{gathered}
$$$$
\mathrm{C}-\mathrm{O}_{[\mathrm{GO}]}+\text { electrons } \rightarrow \mathrm{C}=\mathrm{C}, \mathrm{C}-\mathrm{H}\left(\mathrm{Csp}^{3} \text {, defect carbon }\right)_{[\mathrm{rGO}]}
$$

A second mechanism would involve the reduction of GO with electrons (either photogenerated or supplied upon polarization) without causing the loss of carbon atoms and leading to a partial restoration of the $\mathrm{sp}^{2}$ conjugation and/or the accumulation of other carbon defects (likely $\mathrm{Csp}^{3}$ ). The introduction of defects via hydrogenation of $\mathrm{sp}^{2}$ domains by electrochemical reduction under controlled conditions (e.g., potential, electrolyte) has been reported in our previous study. ${ }^{28}$ As the photochemical reduction proceeds, the nature of the starting GO material gradually changes from $\mathrm{p}$ - to $\mathrm{n}$-type semiconductor, favoring the coexistence of reactions triggered by both charge carriers and allowing the reduction to propagate. These mechanisms can explain the electrochemical behavior of the samples and the Raman and XPS characterization. The predominance of the formation of vacancies in the graphenic sheets upon photochemical reduction would explain the poor electrochemical response manifested in the voltammetric scans of PhrGO, most likely due to the deficient conductivity in the disrupted $\mathrm{sp}^{2}$ network. For ErGO, the mild conditions used during the electrochemical reduction create a material where $\mathrm{Csp}^{3}$ defects are dominant over vacancies; thus ErGO displays larger domains of $\mathrm{sp}^{2}$ network that accounts for a better electrochemical response than PhrGO.

\section{Conclusions}

The reduction of GO thin film electrodes was carried out by combining photochemical and electrochemical approaches, aiming at exploring the influence of such treatments on the surface defects formed on the reduced graphene oxides. Illumination under simulated solar light at low energy flux triggered the reduction of GO following a photochemical reaction pathway and preventing the removal of groups by photothermal effects. The p-type semiconductor character of GO allows the photogeneration of excitons upon irradiation and these charge carriers provoked the reduction of the material. The photochemical reduction brought about the selective removal of epoxide and hydroxyl groups and the resulting material is characterized by fragmented $\mathrm{sp}^{2}$ domains due to a high density of vacancies. In contrast, the electrochemical reduction favored the formation of $\mathrm{sp}^{3}$ defects over vacancies; owing to this, the electroreduced material displayed better electrochemical features than the photoreduced one. When both processes are combined, electrochemical reduction carried out first prevented the formation of vacancies upon subsequent illumination. When the treatment was reversed, both types of defects accumulated in the rGO material. These results showed that a combined photochemical and electrochemical reduction allows the selective removal of O-groups in GO, while controlling the nature of the defects introduced to the basal planes of the graphene sheets. Both factors are crucial when tuning the properties (e.g., reactivity, stability, and conductivity) of rGO materials for a particular application.

\section{Methods}

\section{Materials}

Powdered graphite (98 wt\% purity, Sigma Aldrich) was oxidized by a Hummers' method described elsewhere. ${ }^{62}$ Briefly, $2 \mathrm{~g}$ of 
graphite were mixed with $46 \mathrm{~mL}$ of sulfuric acid (98\%, Sigma Aldrich), $1.0 \mathrm{~g}$ of sodium nitrate (98 wt\% purity, Sigma Aldrich), $6 \mathrm{~g}$ of potassium permanganate (98 wt\% purity, Sigma Aldrich) and $276 \mathrm{~mL}$ of hydrogen peroxide at 3\% v/v (Sigma Aldrich). The resulting mixture was extensively washed with distilled water and dried at $60{ }^{\circ} \mathrm{C}$. As-prepared graphite oxide was suspended in water ( $c a .10 \mathrm{mg} \mathrm{L}^{-1}$ ) and was exfoliated by sonication for $4 \mathrm{~h}$; the suspension was centrifuged ( $c a .4500 \mathrm{rpm}$, $1 \mathrm{~h}$ ) and the graphene oxide sheets were collected from the solution with a concentration of $c a .1 \mathrm{mg} \mathrm{mL}{ }^{-1}$. The bulk GO was characterized to determine the extent of the oxidation and the nature of O-groups before the preparation of the electrodes.

\section{Preparation of GO and rGO film electrodes}

GO thin films were prepared by drop casting the GO aqueous suspensions onto conductive ITO glass substrates (Sigma-Aldrich), previously cleaned in an ultrasonic bath using ethanol, and rinsed with a mixture of ethanol/acetone. About $100 \mu \mathrm{L}$ of the GO suspension (equivalent to $0.1 \mathrm{mg}$ of GO) were added dropwise on the substrate, allowed to dry at $80{ }^{\circ} \mathrm{C}$ for $1 \mathrm{~h}$ and then at room temperature overnight. The obtained GO films were stored in a desiccator until use. The electrochemical reduction of the GO films was performed in a standard three-electrode system using an electrochemical cell with an optically flat circular quartz window (diameter $2 \mathrm{~cm}$ ). The GO films were used as the working electrode, and $\mathrm{Pt}$ wire and $\mathrm{Ag} / \mathrm{AgCl}$ (saturated $\mathrm{KCl}$ ) were used as the counter and reference electrodes, respectively. Briefly, the prepared GO films were immersed in an aqueous solution containing $0.6 \mathrm{mM}$ $\mathrm{NaCl}$ as the inert electrolyte. Unless otherwise stated, all the potentials reported will be referred to the $\mathrm{Ag} / \mathrm{AgCl}$ (saturated $\mathrm{KCl}$ ) reference system. The electrochemical reduction was carried out under mild conditions by cyclic voltammetry ( $c a$. 10 scans) between +0.6 and $-1 \mathrm{~V}$ in a potentiostat/galvanostat (VMP3 Bio-Logic) at $2 \mathrm{mV} \mathrm{s}^{-1}$. The photochemical reduction was performed by frontside illumination of the electrodes for 6 hours under open circuit conditions (no bias potential) using unfiltered simulated solar light (ScienceTech SLB-150B simulator, emitting between 200 and $800 \mathrm{~nm}$ ) positioned at $80 \mathrm{~mm}$ of the electrode The intensity of the illumination delivered at the electrodes was measured using a standard silicon photodiode (Thorlabs). Combined photochemical and electrochemical reduction processes were carried out by sequentially exposing the GO films to electrochemical polarization followed by $6 \mathrm{~h}$ illumination, and vice versa (i.e., $6 \mathrm{~h}$ illumination followed by electrochemical polarization as indicated above). After the reduction, the film electrodes were labelled as XrGO or XYrGO, where $\mathrm{X}$ and $\mathrm{Y}$ account for the first and second reduction method, respectively: electrochemical (E) and photochemical (Ph). For instance, electrode EPhrGO refers to the GO film exposed to electrochemical reduction followed by $6 \mathrm{~h}$ of illumination. All the reduction treatments were performed several times using various electrodes to evaluate the reproducibility; average values corresponding to at least 2 electrodes are presented.

\section{Characterization techniques}

X-ray diffraction patterns were recorded between 5 and $90(2 \theta)$ degrees using a diffractometer (Bruker instrument, D8 Advance) equipped with $\mathrm{Cu} \mathrm{K} \alpha(0.15406 \mathrm{~nm})$ radiation, an energy discriminator detector (SOLX) and operating at $340 \mathrm{kV}$ and $40 \mathrm{~mA}$. Temperature-programmed desorption was carried out using a thermobalance (Netzsch). About $5 \mathrm{mg}$ of pristine GO were placed in an alumina crucible, and heated at $10{ }^{\circ} \mathrm{C} \mathrm{min}^{-1}$ under an argon flow ( $c a .100 \mathrm{~mL} \mathrm{~min}{ }^{-1}$ ) between 25 and $900{ }^{\circ} \mathrm{C}$. The composition of the evolved gases was analyzed and quantified using a mass spectrometer (Pfeiffer). Spectral deconvolution was performed by using a multiple Gaussian function and the Levenberg-Marquardt iteration method. The optical properties of the films were determined by UV-vis spectroscopy in a spectrophotometer (Shimadzu UV-2501, Kyoto, Japan) equipped with an integrating sphere and operating in absorption mode, with $\mathrm{BaSO}_{4}$ as the reference. Spectra were recorded between 400 and $700 \mathrm{~nm}$, due to the cut-off limit of the ITO glass substrate. X-ray photoelectron spectroscopy (XPS) experiments were performed using a $\mathrm{PHI}$ 5000 VersaProbe II apparatus (Physical Electronics) using monochromatic Al-K $\alpha(1486.6 \mathrm{eV})$ radiation and a dual beam charge neutralizing system. The spectra of the dried films were recorded using a $100 \mu \mathrm{m}$ beam size, operating at $25 \mathrm{~W}$ and $15 \mathrm{kV}$, and using a pass energy of $23.5 \mathrm{eV}$ and a $0.2 \mathrm{eV}$ step size for 50 sweeps. Semi-quantitative elemental surface analyses were performed using the survey spectra with the corresponding sensitivity factors. Processing of the collected spectra was performed using energy values referenced to the $\mathrm{C} 1 \mathrm{~s}$ peak of adventitious carbon located at $284.6 \mathrm{eV}$, and a Shirley-type background function. ${ }^{63}$ The deconvolution of the spectra was performed using MultiPak 9.3 software, using a Gaussian (90\%)-Lorentzian (10\%) peak shape by combining the different components and minimizing the total square-error. The spectra show $\mathrm{C}\left(\mathrm{sp}^{2}\right)$ with a binding energy at $284.6 \pm 0.2 \mathrm{eV}, \mathrm{C}\left(\mathrm{sp}^{3}\right)$ at $285.5 \pm 0.2 \mathrm{eV}, \mathrm{C}-\mathrm{O}$ at $286.7 \pm$ $0.2 \mathrm{eV}$ (hydroxyls and epoxides), $\mathrm{C}=\mathrm{O}$ at $287.5 \pm 0.2 \mathrm{eV}$ (ketones, aldehydes and quinones) and $\mathrm{COOH}$ at $288.9 \pm 0.2 \mathrm{eV}$ (carboxylic acids, anhydrides and esters).

The full width at half maximum (FWHM) of each peak was maintained below $1.8 \mathrm{eV}$ in all cases. Raman spectra were recorded under ambient conditions in a Renishaw InVia Qontor spectrometer equipped with an $\mathrm{Ar}^{+}$laser source $(2.41 \mathrm{eV} /$ $514.5 \mathrm{~nm})$. The spectra were collected using a Leica DM2500 optical microscope with a $50 \times$ long objective. The scattered Raman light was dispersed by a holographic grating of 600 grooves per $\mathrm{mm}$, in order to acquire the whole range of interest for carbons $\left(500-5000 \mathrm{~cm}^{-1}\right)$. Each spectrum was recorded with an integration time of $5 \mathrm{~s}$; data presented represent the average of three measurements. The quantitative Raman parameters were obtained from the deconvolution of Raman spectra by a classical Gaussian/Lorentzian fitting process using a linear baseline. Light elements (carbon, oxygen and nitrogen) in the thin films as well as their thickness were quantified by Nuclear Reaction Analysis (NRA) technique at CEMHTI Pelletron accelerator (http://emir.in2p3.fr/CEMHTI) with a $900 \mathrm{keV} \mathrm{D}^{+}$incident beam $\left(2 \times 2 \mathrm{~mm}^{2}\right)$. The nuclear reactions selected for these investigations were ${ }^{12} \mathrm{C}\left(\mathrm{d}, \mathrm{p}_{0}\right){ }^{13} \mathrm{C}$, ${ }^{16} \mathrm{O}\left(\mathrm{d}, \alpha_{0}\right){ }^{14} \mathrm{~N}$ and ${ }^{14} \mathrm{~N}\left(\mathrm{~d}, \mathrm{p}_{5}\right){ }^{15} \mathrm{~N}$. The $\mathrm{p}_{0}, \alpha_{0}$ and $\mathrm{p}_{5}$ particles were detected at $166^{\circ}$ by using a $25 \mathrm{~mm}^{2}$ passivated implanted planar silicon detector. Carbon, oxygen and nitrogen concentrations in 
atomic percent were extracted from NRA spectra using SIMNRA software. ${ }^{64}$ The beam incidence angle was set at $50^{\circ}$ to the sample normal, allowing the obtainment of depth resolutions of $15 \mathrm{~nm}(\mathrm{O})$ and $40 \mathrm{~nm}(\mathrm{C}, \mathrm{N})$. The probed depth was close to $1 \mu \mathrm{m}$.

\section{Conflicts of interest}

There are no conflicts to declare.

\section{Acknowledgements}

This work was funded by the European Council Research through a Consolidator Grant (ERC-CoG-648161, PHOROSOL) and CONACyT (project SEP-CONACyT, 169634). AQR thanks CONACyT for his PhD scholarship (grant 25840) to perform an internship in France. The authors would like to thank Dr Ammar for helpful discussions.

\section{Notes and references}

1 D. R. Dreyer, S. Park, C. W. Bielawski and R. S. Ruoff, The chemistry of graphene oxide, Chem. Soc. Rev., 2010, 39, 228-240.

2 S. Pei and H. M. Cheng, The reduction of graphene oxide, Carbon, 2012, 50, 3210-3228.

3 S. Stankovich, D. A. Dikin, R. D. Piner, K. A. Kohlhaas, A. Kleinhammes, Y. Jia, Y. Wu, S. Binh, T. Nguyen and R. S. Ruoff, Synthesis of graphene-based nanosheets via chemical reduction of exfoliated graphite oxide, Carbon, 2007, 45, 1558-1565.

4 Y. Shen, T. Jing, W. Ren, J. Zhang, Z. G. Jiang, Z. Z. Yu and A. Dasari, Chemical and thermal reduction of graphene oxide and its electrically conductive polylactic acid nanocomposites, Compos. Sci. Technol., 2012, 72, 1430-1435.

5 M. Acik, G. Lee, C. Mattevi, A. Pirkle, R. M. Wallace, M. Chhowalla, K. Cho and Y. Chabal, The Role of Oxygen during Thermal Reduction of Graphene Oxide Studied by Infrared Absorption Spectroscopy, J. Phys. Chem. C, 2011, 115, 19761-19781.

6 X. Gao, J. Jang and S. Nagase, Hydrazine and Thermal Reduction of Graphene Oxide: reaction Mechanisms and Design, J. Phys. Chem. C, 2010, 114, 832-842.

7 P. V. Kumar, N. M. Bardhan, G. Chen, Z. Li, A. M. Belcher and J. M. Grossman, New insights into the thermal reduction of graphene oxide: Impact of oxygen clustering, Carbon, 2016, 100, 90-98.

8 E. Tegou, G. Pseiropoulos, M. K. Filippidou and S. Chatzandroulis, Low-temperature thermal reduction of graphene oxide films in ambient atmosphere: Infra-red spectroscopic studies and gas sensing applications, Microelectron. Eng., 2016, 159, 146-150.

9 A. Ambrosi and M. Pumera, Precise tuning of surface composition and electron-transfer properties of graphene oxide films through electroreduction, Chem. - Eur. J., 2013, 19, 4748-4753.
10 J. Kauppila, P. Kunnas, P. Damlin, A. Viinikanoja and C. Kvarnström, Electrochemical reduction of graphene oxide films in aqueous and organic solutions, Electrochim. Acta, 2013, 89, 84-89.

11 A. Viinikanoja, Z. Wang, J. Kauppila and C. Kvarnström, Electrochemical reduction of graphene oxide and its in situ spectroelectrochemical characterization, Phys. Chem. Chem. Phys., 2012, 14, 14003-14009.

12 R. Backreedy, J. M. Jones, M. Pourkashanian and A. Williams, A study of the reaction of oxygen with graphite, J. Chem. Inf. Model., 2001, 119, 385-394.

13 A. Bagri, C. Mattevi, M. Acik, J. Y. Chabal, M. Chhowalla and V. B. Shenoy, Structural evolution during the reduction of chemically derived graphene oxide, Nat. Chem., 2010, 2, 581-587.

14 Y. Matsumoto, M. Koinuna, S. Ida, S. Hayami, T. Taniguchi, K. Hatakeyama, H. Tateishi, Y. Watanabe and S. Amano, Photoreaction of Graphene Oxide Nanosheets in Water, J. Phys. Chem. C, 2011, 115(39), 19280-19286.

15 R. Y. N. Gengler, D. S. Badali, D. Zhang, K. Dimos, K. Spyrou, D. Gournis and R. J. D. Miller, Revealing the ultrafast process behind the photoreduction of graphene oxide, Nat. Commun., 2013, 4, 2560.

16 M. Mohandoss, S. S. Gupta, A. Nelleri, T. Pradeep and S. M. Maliyekkal, Solar mediated reduction of graphene oxide, RSC Adv., 2017, 7, 957-963.

17 H. Öztürk, D. Ekinci and Ü. Demir, Atomic scale imaging and spectroscopic characterization of electrochemically reduced graphene oxide, Surf. Sci., 2013, 611, 54-59.

18 J. D. Roy-mayhew and I. A. Aksay, Graphene Materials and Their Use in Dye-Sensitized Solar Cells, Chem. Rev., 2014, 114, 6323-6348.

19 D. Lopez-Diaz, M. Lopez Holgado, J. L. Garcia-Fierro and M. M. Velazquez, Evolution of the Raman Spectrum with the Chemical Composition of Graphene Oxide, J. Phys. Chem. C, 2007, 121, 20489-20497.

20 K. Hatakeyama, K. Awaya, M. Koinuma, Y. Shimizu, Y. Hakuta and Y. Matsumoto, Production of water-dispersible reduced graphene oxide without stabilizers using liquid-phase photoreduction, Soft Matter, 2017, 13(45), 8353-8356.

21 S. Eigler, C. Dotzer, A. Hirsch, M. Enzelberger and P. Müller, Formation and Decomposition of $\mathrm{CO}_{2}$ Intercalated Graphene Oxide, Chem. Mater., 2012, 24(7), 1276-1282.

22 M. P. Araujo, O. S. G. P. Soares, A. J. S. Fernandes, M. F. R. Pereira and C. Freire, Tuning the surface chemistry of graphene flakes: new strategies for selective oxidation, RSC Adv., 2017, 7, 14290-14301.

23 A. B. Dongil, B. Bachiller-Baeza, A. Guerrero-Ruiz, I. Rodríguez-Ramos, A. Martínez-Alonso and J. M. D. Tascón, Surface chemical modifications induced on high surface area graphite and carbon nanofibers using different oxidation and functionalization treatments, J. Colloid Interface Sci., 2011, 355, 179-189.

24 S. L. Kuo, W.-R. Liu, C.-P. Kuo, N.-L. Wu and H.-C. Wu, Lithium storage in reduced graphene oxides, J. Power Sources, 2013, 244, 552-556. 
25 M. Acik, G. Lee, C. Mattevi, M. Chhowalla, K. Cho and Y. J. Chabal, Unusual infrared-absorption mechanism in thermally reduced graphene oxide, Nat. Mater., 2010, 9, 840-845.

26 K. Erickson, R. Erni, Z. Lee, N. Alem, W. Gannett and A. Zettl, Determination of the local chemical structure of graphene oxide and reduced graphene oxide, Adv. Mater., 2010, 22, 4467-4472.

27 H. Tong, J. Zhu, J. Chen, Y. Han, S. Yang, B. Ding and $\mathrm{X}$. Zhanget, Electrochemical reduction of graphene oxide and its electrochemical capacitive performance, J. Solid State Electrochem., 2013, 17, 2857-2863.

28 J. A. Quezada-Renteria, C. O. Ania, L. F. Chazaro-Ruiz and J. R. Rangel-Mendez, Influence of protons on reduction degree and defect formation in electrochemically reduced graphene oxide, Carbon, 2019, 149, 722-732.

29 W. J. Basirun, M. Sookhakian, S. Baradaran, M. R. Mahmoudian and M. Ebadi, Solid-phase electrochemical reduction of graphene oxide films in alkaline solution, Nanoscale Res. Lett., 2013, 8, 397.

30 V. Abdelsayed, S. Moussa, M. H. Hassan, H. S. Alurri, M. M. Collison and M. S. El-Shall, Photothermal Deoxygenation of Graphite Oxide with Laser Excitation in Solution and Graphene-Aided Increase in Water Temperature, J. Phys. Chem. Lett., 2010, 1(19), 2804-2809.

31 L. Huang, Y. Liu, L.-C. Ji, Y.-Q. Xie, T. Wang and W.-Z. Shi, Pulsed laser assisted reduction of graphene oxide, Carbon, 2011, 49, 2431-2436.

32 P. S. Guin, S. Das and P. C. Mandal, Electrochemical Reduction of Quinones in Different Media: A Review, Int. J. Electrochem., 2011, 816202.

33 X. Zhang, D. Zhang, Y. Chen, X. Sun and Y. Ma, Electrochemical reduction of graphene oxide films: Preparation, characterization and their electrochemical properties, Chin. Sci. Bull., 2012, 57, 045-3050.

34 Y.-Q. Liu, Y.-L. Zhang, Y. Liu, H.-B. Jiang, D.-D. Han, B. Han, J. Feng and H.-B. Sun, Surface and Interface Engineering of Graphene Oxide Films by Controllable Photoreduction, Chem. Rec., 2016, 16, 1244-1255.

35 J. I. Paredes, S. Villar-Rodil, A. Martínez-Alonso and J. M. D. Tascón, Graphene oxide dispersions in organic solvents, Langmuir, 2008, 24, 10560-10564.

36 D. Li, M. B. Muller, S. Gilje, R. B. Kaner and G. G. Wallace, Processable aqueous dispersions of graphene nanosheets, Nat. Nanotechnol., 2008, 3, 101-105.

37 L. Guardia, S. Villar-Rodil, J. I. Paredes, R. Rozada, A. Martinez-Alonso and J. M. D. Tascon, UV light exposure of aqueous graphene oxide suspensions to promote their direct reduction, formation of graphene-metal nanoparticle hybrids, Carbon, 2012, 50, 1014-1024.

38 A. Ganguly, S. Ganguly, S. Sharma, P. Papakonstantinou and J. Hamilton, Probing the thermal deoxygenation of graphene oxide using high-resolution In Situ X-ray-based spectroscopies, J. Phys. Chem. C, 2011, 115, 17009-17019.

39 C. Botas, P. Álvarez, P. Blanco, M. Granda, C. Blanco, R. Santamaría, L. J. Romasanta, R. Verdejo, M. A. LópezManchado and R. Menéndez, Graphene materials with different structures prepared from the same graphite by the Hummers' and Brodie methods, Carbon, 2013, 65, 156-164.

40 Y. Zhou, Q. Bao, L. A. L. Tang, Y. Zhong and K. P. Loh, Hydrothermal dehydration for the "green" reduction of exfoliated graphene oxide to graphene and demonstration of tunable optical limiting properties, Chem. Mater., 2009, 21(13), 2950-2956.

41 A. Cuesta, P. Dhamelincourt, J. Laureyns, A. MartínezAlonso and J. M. D. Tascon, Raman Microprobe Studies on Carbon Materials, Carbon, 1994, 32, 1523-1532.

42 T. Jawhari, A. Roid and J. Casado, Raman Spectroscopic Characterization of some Commercially Available Carbon Black Materials, Carbon, 1995, 33, 1561-1565.

43 S. Claramunt, A. Varea, D. López-Díaz, M. M. Velázquez, A. Cornet and A. Cirera, The Importance of Interbands on the Interpretation of the Raman Spectrum of Graphene Oxide, J. Phys. Chem. C, 2015, 119, 10123-10129.

44 S. Vollebregt, R. Ishihara, F. D. Tichelaar, Y. Hou and C. I. M. Beenakker, Influence of the Growth Temperature on the First and Second-order Raman Band Ratios and Widths of Carbon Nanotubes and Fibers, Carbon, 2012, 50, 3542-3554.

45 L. Cançado, A. Jorio and M. Pimenta, Measuring the absolute Raman cross section of nanographites as a function of laser energy and crystallite size, Phys. Rev. B: Condens. Matter Mater. Phys., 2007, 76(6), 064304.

46 A. C. Ferrari and J. Robertson, Interpretation of Raman spectra of disordered and amorphous carbon, Phys. Rev. B: Condens. Matter Mater. Phys., 2000, 61, 14095.

47 F. Tuinstra and J. L. Koenig, Raman Spectrum of Graphite, J. Chem. Phys., 1970, 53, 1126-1130.

48 X. Díez-Betriu, S. Álvarez-García, C. Botas, P. Álvarez, J. Sánchez-Marcos, C. Prieto, R. Menéndez and A. de Andrés, Raman spectroscopy for the study of reduction mechanisms and optimization of conductivity in graphene oxide thin films, J. Mater. Chem. C, 2013, 1, 6905-6912.

49 K. N. Kudin, B. Ozbas, H. C. Schniepp, R. K. Prud'homme, I. A. Aksay and R. Car, Raman Spectra of Graphite Oxide and Functionalized Graphene Sheets, Nano Lett., 2008, 8, 36-41.

50 E. del Corro, M. Taravillo and V. G. Baonza, Stress-Dependent Correlations for Resonant Raman Bands in Graphite with Defects, J. Raman Spectrosc., 2014, 45, 476-480.

51 P. Venezuela, M. Lazzeri and F. Mauri, Theory of DoubleResonant Raman Spectra in Graphene: Intensity and Line Shape of Defect-Induced and Two-Phonon Bands, Phys. Rev. B: Condens. Matter Mater. Phys., 2011, 84, 035433.

52 C. Cong, T. Yu, R. Saito, G. F. Dresselhaus and M. S. Dresselhaus, Second-Order Overtone and Combination Raman Modes of Graphene Layers in the Range of 1690$2150 \mathrm{~cm}^{-1}$, ACS Nano, 2011, 5, 1600-1605.

53 G. Williams, B. Seger and P. V. Kamat, $\mathrm{TiO}_{2}-$ graphene nanocomposites. UV-assisted photocatalytic reduction of graphene oxide, ACS Nano, 2008, 2, 1487-1491.

54 H. Li, S. Pang, X. Feng, K. Müllen and C. Bubeck, Polyoxometalate assisted photoreduction of graphene oxide and its nanocomposite formation, Chem. Commun., 2010, 46, 6243-6245. 
$55 \mathrm{~W}$. Chen and L. Yan, Preparation of graphene by a lowtemperature thermal reduction at atmospheric pressure, Nanoscale, 2010, 2, 559-563.

56 S. Urana, A. Alhani and C. Silva, Study of ultraviolet-visible absorvance of exfolidated graphite forms, AIP Adv., 2017, 7, 035323.

57 D.-T. Phan and G.-S. Chung, P-n junction characteristics of graphene oxide and reduced graphene oxide on n-type Si(111), J. Phys. Chem. Solids, 2013, 74, 1509-1514.

58 T. V. Cuong, H. N. Tien, V. H. Luan, V. H. Pham, J. S. Chung, D. H. Yoo, S. H. Hahn, K.-K. Koo, P. A. Kohl, S. H. Hur and E. J. Kim, Solution-processed semitransparent $\mathrm{p}-\mathrm{n}$ graphene oxide:CNT/ZnO heterojunction diodes for visible-blind UV sensors, Phys. Status Solidi A, 2011, 208, 943-946.

59 N. Dien, K. Tu, J. Choi, C. R. Park and H. Kim, Remarkable Conversion Between n- and p-Type Reduced Graphene Oxide on Varying the Thermal Annealing Temperature, Chem. Mater., 2015, 27, 7362-7369.

60 A. Bhaumik and J. Narayana, Conversion of p to n-type reduced graphene oxide by laser annealing at room temperature and pressure, J. Appl. Phys., 2017, 121, 125303.

61 Y. Y. Gurevich and Y. V. Pleskov, Photoelectrochemistry of Semiconductors, in Semiconductors and Semimetals, ed. R. K. Willardson, A. C. Beerpp, Academic Press Inc., Elsevier Ltd, 1983, ch. 4, vol. 19, pp. 255-328.

62 W. W. Hummers and R. E. Offeman, Preparation of graphitic oxide, J. Am. Chem. Soc., 1958, 80(6), 1339.

63 C. D. Wagner, W. M. Riggs, L. E. Davis and J. F. Moulder, Handbook of X-ray photoelectron spectroscopy, PerkinElmer, 1979.

64 M. Mayer, SIMNRA user's guide, Technical Report IPP 9/113, Max-Planck-Institute für Plasmaphysik, Garching, 1997. 